\title{
$O$ enfermeiro e o ensino na assistência às doenças sexualmente transmissíveis: revisão integrativa
}

\section{Nurses and teaching in assistance for sexually transmitted diseases: a integrative review}

\author{
João Carlos Alves dos Santos \\ Enfermeiro graduado pela UNICERP/MG. Especialista em docência em saúde FAMERP/SP. Especializando em \\ Urgência e emergência e UTI geral na UNIRP/SP.
}

\begin{abstract}
Resumo - As doenças sexualmente transmissíveis e a síndrome da imunodeficiência humana são doenças de alta prevalência, além de ser um problema de política pública de saúde do país. O objetivo deste estudo foi analisar na literatura nacional estudos a respeito da formação do enfermeiro docente e o seu ensino por meio de métodos e práticas pedagógicas na assistência a essas doenças. Trata-se de um estudo de revisão integrativa. A busca foi realizada na base de dados Literatura Latino-Americana e do Caribe em Ciências da Saúde, mediante a combinação dos descritores: Síndrome da Imunodeficiência Adquirida, Docentes, Ensino, Enfermagem entre o ano 2000 à 2014. Foram encontrados cinco artigos após a aplicação dos critérios de inclusão pré-definidos. Observou-se o relato de experiência e a valorização da percepção pessoal e profissional como instrumentos que alicerçam o ensino ao tema pelos docentes de enfermagem. Portanto, sugere-se a ampliação de pesquisas sobre o contexto docente e as estratégias de ensino, pois estudos com esse enfoque são fundamentais para uma percepção reflexiva do ensino em enfermagem e contribui para a melhoria de sua ação como educador.
\end{abstract}

Palavras-chaves - AIDS; Docentes; Ensino; Enfermagem.

\begin{abstract}
Sexually transmitted diseases and human immunodeficiency syndrome are highly prevalent diseases. Also, they are a public policy issue of health of the country. The aim of this study was to analyze in the national literature studies on the training of teaching nurses and their education through educational methods and practices in the care of these diseases. This is a study of integrative review. The search was performed in the database Latin American and Caribbean Health Sciences. Through the combination of descriptors: Human Immunodeficiency Syndrome, Teachers, Education, Nursing between 2000 and 2014. Five articles were found after application of predefined inclusion criteria. We observed a report of experience and the enhancement of personal and professional perception as instruments that support teaching on the subject by nursing professors. Therefore, we suggest the expansion of research on the teaching context and teaching strategies, because studies with this approach are key to a reflexive perception of nursing education and they contribute to the improvement of its action as an educator.
\end{abstract}

Key words - AIDS; Education; Teachers; Nursing. 


\section{INTRODUÇÃO}

A Organização Mundial de Saúde (OMS) estima que de 1990 a 2008, houvesse um aumento de 99, 6\% de casos de Doenças Sexualmente Transmissíveis (DST) curáveis na faixa etária de 15 a 49 anos. A OMS ainda estimou de 10 a 12 milhões de casos por ano de DST curáveis no Brasil e outros milhões de DST não curáveis produzindo altíssimo impacto na saúde da população devido as suas consequências, tais como infertilidade, abortos espontâneos, natimortos, infecções congênitas e outros (OMS, 2012).

De 2000 a 2012, foram notificados no estado de São Paulo 58.402 casos de sífilis adquirida e síndrome uretral masculino (SÃO PAULO, 2012). Ambas as doenças aumentam a exposição ao Vírus da Imunodeficiência Humana (HIV) e sua transmissão (BRASIL, 2014). Por sua vez, existe estimativa mundial de cerca de 42 milhões de pessoas infectadas com o HIV (UNAIDS, 2012). A Síndrome da Imunodeficiência Adquirida (AIDS) no Brasil continua em patamares elevados, com 757.042 casos registrados de 1980 a 2014 e com uma taxa de incidência oscilando em torno de 20,5 casos por 100 mil habitantes (BRASIL, 2014).

A abordagem das pessoas com DST/AIDS envolve uma particularidade que a torna complexa que é a sexualidade que envolve tabus, preconceitos, desinformações e informações incorretas. Proporcionalmente, a ausência de reflexões no âmbito familiar e escolar contribui para a sua estigmatização.

A partir disto, os interesses sobre o assunto provem da minha atual função de aconselhador em DST/AIDS e Hepatites B e C e de ser Multiplicador de Testes Rápidos.

A relevância científica e social define-se em como o docente utiliza-se de seus saberes pessoais e profissionais para tratar e produzir conhecimento sobre o tema.

Nessa perspectiva, o objetivo deste estudo é analisar o que tem sido produzido na literatura nacional sobre o docente de enfermagem em sua ação educadora de profissionais de enfermagem no ensino no tocante em como abordar/cuidar dos pacientes acometidos pelas DST/AIDS.

\section{MATERIAL E MÉTODOS}

Trata-se de um estudo exploratório e descritivo com abordagem quantitativa e qualitativa. A revisão de literatura de pesquisa tem como propósito reunir conhecimentos sobre um tema. Permite ainda que o leitor compreenda os antecedentes do conhecimento atual sobre um assunto e elucida a importância de um novo estudo (POLIT, BECK, 2011).

Além disso, a revisão integrativa é um método de revisão mais amplo, pois permite incluir literatura teórica e empírica bem como estudos com diferentes abordagens metodológicas quantitativa e qualitativa (URSI, 2005; SOUZA et al., 2010).

A revisão integrativa reúne e sintetiza os estudos realizados sobre um determinado tema, construindo uma conclusão, a partir dos resultados evidenciados em cada estudo, mas que investiguem problemas idênticos ou similares (SOUZA et al., 2010).

Rev. Bra. Edu. Saúde v. 6, n.2, p. 08-12, 2016
Para tanto, a revisão integrativa utiliza-se de seis fases que compõem esse processo: estabelecimento da hipótese e objetivos da revisão integrativa; estabelecimento de critérios de inclusão e exclusão de artigos (seleção da amostra); definição das informações a serem extraídas dos artigos selecionados; análise dos resultados; discussão e apresentação dos resultados e apresentação da revisão (URSI, 2005; POMPEO et al., 2009 ; SOUZA et al., 2010).

Neste sentido, é necessário para a análise e avaliação dos resultados e apoio para discussão elaborar um quadro para auxiliar a exploração dos artigos com perguntas às fontes do estudo (URSI, 2005). A revisão integrativa é um método que permite gerar uma fonte de conhecimento atual sobre o problema e determinar se o conhecimento é válido para ser transferido para a prática; deve seguir padrões de rigor metodológico e oferecer subsídios para o avanço da pratica em saúde (URSI, 2005; SOUZA et al., 2010).

Autores apontam que a maioria das bases de dados eletrônicas de interesse para os enfermeiros pode ser acessada pela busca online (POLIT, BECK, 2011). Logo, foi utilizada a base de dados da Literatura LatinoAmericana e do Caribe em Ciências da Saúde (LILACS) da Biblioteca Virtual em Saúde do Centro LatinoAmericano e do Caribe de Informação em Ciências da Saúde (BIREME). Pois, trate-se de uma fonte de consultas no Brasil e reuni um grande número de estudos brasileiros (POLIT, BECK, 2011)

A busca ocorreu até 30 Abril de 2014, mediante a combinação dos descritores "(Docentes ou Ensino) e (DSTs ou HIV ou AIDS) e (enfermagem)". Ao realizar o cruzamento dos descritores, inicialmente foram encontradas 1.345 referências bibliográficas, e foram excluídos os estudos que não preencheram os critérios de inclusão.

Os critérios de inclusão dos artigos selecionados para amostra desta revisão foram: disponíveis no banco de dados LILACS, publicados em língua portuguesa; com resumos disponíveis via online; compreendidos entre 01 de Janeiro de 2000 até Abril 2014 e, por conseguinte, estudos relacionando docentes de enfermagem e o ensino no atendimento as DST/AIDS.

Então, foi efetuada a busca e a leitura na integra dos cinco artigos no próprio banco de dados LILACS. Um formulário adaptado foi utilizado para análise.

\section{RESULTADOS E DISCUSSÃO}

Todos os artigos preencheram o nível de evidência derivada de um único artigo descritivo ou qualitativo e, adiante, apresentar-se-á um panorama geral dos artigos avaliados.

Observou-se que os periódicos que publicaram artigos sobre a referida temática em estudo foi a Revista Brasileira de Enfermagem; Revista Gaúcha de Enfermagem; Revista da Escola de Enfermagem da USP, Escola Anna Nery Revista de Enfermagem e o Jornal Brasileiro de Doenças Sexualmente Transmissíveis, sendo um artigo por revista. Nota-se, assim, um maior número de publicações em revistas de enfermagem (Quadro 1).

Em relação à síntese dos dados verificaram-se importantes contribuições levantadas pelos os autores dos artigos revisados (Quadro 2). 
Quadro 1- Distribuição da caracterização das publicações dos artigos revisados de 2001 a 2013. São José do Rio Preto, 2014.

\begin{tabular}{|lllll|}
\hline $\begin{array}{l}\text { Instituição sede do } \\
\text { estudo }\end{array}$ & Tipo de Revista & Ano de Publicação & $\begin{array}{l}\text { Ordem dos } \\
\text { trabalhos revisados } \\
\text { Trabalho 1 }\end{array}$ & $\begin{array}{l}\text { Estado de origem } \\
\text { do estudo } \\
\text { São Paulo }\end{array}$ \\
$\begin{array}{l}\text { USP } \\
\begin{array}{l}\text { Universidade da } \\
\text { Universidade }\end{array}\end{array}$ & Especializada & 2001 & Trabalho 2 & $\begin{array}{l}\text { São Paulo } \\
\text { Trabalho 3 }\end{array}$ \\
$\begin{array}{l}\text { UFPB } \\
\begin{array}{l}\text { Universidade } \\
\text { UFRJ }\end{array}\end{array}$ & $\begin{array}{l}\text { Enfermagem Geral } \\
\text { Secretaria do Estado } \\
\text { de Saúde - SP }\end{array}$ & 2003 & Trabalho 4 & Rio de Janeiro \\
\hline
\end{tabular}

Quadro 2 - Apresentação da síntese do artigo incluído na revisão integrativa. São José do Rio Preto, 2014.

Nome da pesquisa
Pesquisa-Ação sobre sexualidade, DST/AIDS com
enfermeiros professores de escola de enfermagem
(MIYASAKI, BUENO, 2001) ${ }^{1}$.
Resultados
Os entrevistados apresentam a sua percepção de vida no
âmbito individual e coletivo, valores humanos e morais de
forma satisfatória. Evidenciam a educação sexual nas
escolas, universidades e família como ainda despreparados
para lidarem com esta questão.

\section{Nome da pesquisa}

Relato de uma experiência de ensino de enfermagem em saúde coletiva: a informática no ensino de Vigilância Epidemiológica (NICHIATA, et al., 2003)².

\section{Resultados}

Os alunos desenvolveram a habilidade de usar o banco de dados Epi-info. Os alunos expressaram sua compreensão sobre os problemas de saúde da população estudada com AIDS. Os docentes assumiram um papel de mediadores.

\section{Nome da pesquisa}

Relato de experiência: Abordagem por Competências no Processo ensino-aprendizagem (SILVA et al., 2005) ${ }^{3}$.

\section{Resultados}

Falta de conhecimento sobre a legislação vigente, sobre os direitos e deveres do portador de HIV/AIDS, conhecer as Recomendações do Ministério da Saúde sobre aconselhamento.

\section{Nome da pesquisa}

Teoria e prática do docente sobre HIV/AIDS na graduação em enfermagem (LIMA et al., 2005) ${ }^{4}$.

\section{Resultados}

Os docentes relatam limitadores na inserção do conteúdo no programa de ensino. Por outro lado, o professor pode colocar os conteúdos de PNDST/AIDS de três formas: no currículo institucional; nas disciplinas e por meio da experiência e a história pessoal do professor.

\section{Nome da pesquisa}

O ensino de enfermagem sobre HIV/AIDS sob a ótica da cidadania (CAMILLO et al., 2013) ${ }^{5}$.

\section{Resultados}

A importância da reflexão e da criticidade do professor no ensino de HIV/AIDS; o exercício da cidadania por meio do ensino e a compreensão do humano no ensino a partir da vivência com o indivíduo com HIV/AIDS.

\section{Objetivo}

Levantar a percepção que os docentes têm sobre a sexualidade DST/AIDS. Trabalhar programas educativos sobre estas temáticas.

\section{Recomendações/conclusões}

Os autores evidenciam a sexualidade e as questões sexuais no âmbito social como estereotipados, mas em transformação para a desmistificação. Os docentes possuem percepção básica e real sobre a AIDS.

\section{Objetivo}

Discutir a importância da informática no ensino da Enfermagem. Relatar a experiência da disciplina com Enfoque nas Doenças Transmissíveis na utilização da informática como um instrumento pedagógico.

Recomendações/conclusões

Os autores assinalam as características instrucionais dos computadores e como propiciam melhores condições de ensino e aprendizagem.

Os autores identificaram a informática como um potente instrumento na vigilância epidemiológica.

\section{Objetivo}

Demonstrar os procedimentos didáticos de uma regência de ensino baseada na abordagem por competências.

\section{Recomendações/conclusões}

Os autores sugerem mais relatos de experiências na literatura, busca de uma identidade da pedagogia por competência e a consequente prática desta produção. Sugerem-se relatos de experiências, relacionada ao significado dos participantes.

\section{Objetivo}

Identificar se os conteúdos de ensino teórico e prático se relacionam com as ações preconizadas no Programa Nacional de DST/AIDS.

\section{Recomendações/conclusões}

As autoras indicam que os professores devem inserir no seu programa de ensino de forma planejada e não por oportunidades e depender da experiência pregressa. Desenvolver atividades de treinamento para enfrentamento da epidemia e a sua educação atualizada.

\section{Objetivo}

Verificar a percepção de docentes de enfermagem sobre a responsabilidade social a temática do HIV/AIDS.

\section{Recomendações/conclusões}

Os autores classificam abordagem ética e cidadã como preponderante para a assistência aos clientes com HIV/AIDS. A vivência com os clientes gera atitudes e percepções necessárias para a profissão e o autocuidado. 
Todos os primeiros autores são enfermeiros, $80 \%$ do sexo feminino, $100 \%$ das instituições sede do estudo foram instituições públicas e somente uma enfermeira assistencial.

O tipo de pesquisa mais frequentes foi à abordagem qualitativa. Por sua vez, a pesquisa com abordagem qualitativa é utilizada pela enfermagem devido a mensuração holística de significados e representação dos sujeitos ao objeto de estudo (OLIVEIRA et al.. 2007; HOLANDA et al., 2013).

Não houve artigos com a participação de graduando de enfermagem sobre o processo de ensino das DST/AIDS e também não foram encontrados artigos de docentes do curso profissionalizante.

$\mathrm{Na}$ maioria dos artigos os autores não apontaram lacunas, limitações e vieses nos seus estudos. Somente um artigo apontou todos eles. Os seus reconhecimentos sugerem novas pesquisas e limitação da mensuração dos dados pelo leitor.

Sobre o rigor metodológico, $80 \%$ das amostras cumpriram quase todas as etapas sugeridas pelo formulário aplicado e $20 \%$ cumpriram todas as etapas.

$\mathrm{O}$ relato de experiência se mostrou um veículo importante para fomentar pesquisas pelos docentes e para publicações e uma ferramenta para a transmissão de experiências sobre métodos pedagógicos que o auxiliam.

Todavia, só um trabalho citou - e de forma generalista - as DSTs. Em contraste com sua importância na saúde pública do país, e ainda mais não houve continuação da investigação pelos autores.

Percebe-se que as publicações sobre os docentes em enfermagem e DST/ AIDS na literatura nacional possuem lacunas e limitações para serem vencidas pelas poucas publicações.

$\mathrm{Na}$ perspectiva de responder ao objetivo formulado nesta revisão, já era esperado que a maioria dos estudos das publicações de revistas de enfermagem geral fosse realizada por docentes mestres e doutores de universidades; o Estado de São Paulo em destaque com instituições sede de estudos (HOLANDA et al., 2013). Quanto à participação dos graduandos de enfermagem em pesquisa sobre o HIV/AIDS, isto foi algo comum encontrado na literatura (HOLANDA et al., 2013). Entretanto, não foi encontrada a mesma participação de graduandos.

Ademais, não houve artigos de revisão sobre o assunto, apesar de ser uma prática de investigação comum na enfermagem (HOLANDA et al., 2013). O enfermeiro docente utiliza-se do conhecimento atual gerado por revisões para fundamentar a melhor decisão sobre o ensino/abordagem de um determinado cuidado de enfermagem (POLIT, BECK, 2011).

A interação dos estudantes com o indivíduo com HIV/AIDS gera oportunidade de análises e discussões sobre as situações próximas, exigindo dele abertura para o novo e para a reflexão se tornando uma atividade de ensino (CAMILO et al., 2013).

A condição de ter uma DST aumenta até 10 vezes a possibilidade de ser infectar pelo HIV. As úlceras genitais sobem para até 18 vezes a transmissibilidade. O portador de HIV que tem uma DST transmite mais facilmente o HIV aos seus parceiros sexuais. Em todos os artigos revisados o termo HIV resultou em sinônimo de AIDS.
Contudo, a AIDS é o estágio mais avançado em que o portador do HIV se encontra (BRASIL, 2014).

Há incipiência de trabalhos nacionais publicados sobre o tema. Apesar disso, os autores dos artigos demonstraram conhecimento aprofundado da temática. É preciso considerar a possibilidade de os enfermeiros publicarem em outros periódicos que não sejam da área específica da enfermagem (ARAÚJO et al., 2005).

Este estudo pode servir de auxilio a literatura, mas carece de maior aprofundamento e novos estudos pela sua complexidade, a não generalizações dos resultados pela realidade temporal da epidemia. Assim, cada vez mais, diminuir a lacuna existente entre o ensino e abordagem mais adequada para a realidade temporal para atender o portador de DST/AIDS.

Indiscutivelmente, devem-se elaborar artigos que discutam assuntos de ordem contemporânea e que forneçam estratégias pedagógicas para o ensino e divulgação dos temas sobre sorodiscordância imunológica entre heterossexuais e homossexuais, redução de danos como estratégia educativa, gestão de risco sexual, a abordagem sindrômica de algumas DSTs que o enfermeiro pode atuar.

O Processo de Enfermagem dever ser utilizado em todos os ambientes e situações de saúde no território nacional (COFEN, 2009). O processo em enfermagem é o instrumento que viabiliza o cuidado e o docente pode usálo no ensino referente às DST/AIDS, além de ser uma estratégia que pode ser pedagógica e que organiza o cuidado e produz conhecimento sobre o tema.

$\mathrm{O}$ ensino com a metodologia ativa proporciona atividades interativas que ajudam na construção do conhecimento em relação à essa temática (CAMILO et al., 2013).

As discussões dos artigos contribuíram para a literatura com abordagens contemporâneas e atualizadas do cuidado, não só biológico e psicológico. Mas também valorizando valores éticos e morais e acrescentando os direitos sociais como parte da ação pedagógica dos docentes no seu ensino, com reflexões existenciais, transcendentais ao mesmo nível das outras competências.

\section{CONCLUSÕES}

A temática sobre os docentes de enfermagem e o ensino das DST/HIV/AIDS é relevante para a área e tem sido abordada em pesquisas nacionais apesar do reduzido número de artigos analisados na amostra em um período de quatorze anos.

A valorização da percepção do docente sobre o HIV/AIDS foi um assunto discutido. O relato de experiência se mostrou uma ferramenta de divulgação de sua atuação docente. A discussão sobre DST foi limitada em detrimento da sua relevância em saúde pública. Temas contemporâneos na prática assistencial na atenção aos portadores de DST/AIDS não foram abordados como objeto de estudo. Todavia, os resultados são relevantes para a literatura e para prática docente.

Diante da questão norteadora desta revisão bibliográfica, fica nítida a necessidade de ampliar as pesquisas que analisem o contexto docente e as estratégias de ensino relativo às DST/AIDS. 
Portanto é necessário intensificar esforços para o desenvolvimento de pesquisas que produzam estratégias, métodos e práticas pedagógicas para evidências mais fortes relativas ao tema investigado.

\section{REFERÊNCIAS BIBLIOGRÁFICAS}

OMS. Organização mundial de saúde. Geneva; 2012. Global incidence and prevalence of selected curable sexually transmitted infections - 2008. Disponível em: http://www.who.int/about/licensing/copyright_form/en/in dex.html. Acesso em: 25 de Agosto de 2014.

SÃO PAULO. Secretaria de Estado da Saúde de São Paulo. Boletim Epidemiológico. DST/AIDS, São Paulo, v. 29, n.1, 2012.

BRASIL, Ministério da Saúde. Departamento de DST, Aids e Hepatites Virais. Brasília (DF); 2014. Disponível em: http://www.aids.gov.br/. Acesso em: 13 de junho de 2014.

UNAIDS. Programa Conjunto das Nações Unidas sobre HIV/AIDS. Relatório sobre a epidemia mundial da AIDS, 2012. Disponível em: http://www.unodc.org /Resumo.pdf. Acesso em: 13 de junho de 2014.

BRASIL. Ministério da Saúde. Boletim epidemiológico AIDS/DST. Brasília: Ministério da Saúde; 2014.

POLIT, D. F.; BECK, C. T. Fundamentos de pesquisa em enfermagem: avaliação de evidências para a prática da enfermagem. 7. ed. Porto Alegre: Artmed; 2011. 670p.

URSI. E. S. Prevenção de lesões de pele no perioperatório: revisão integrativa da literatura [Dissertação]. Ribeirão Preto: Escola de Enfermagem de Ribeirão Preto, Universidade de São Paulo; 2005.128p.

SOUZA, M. T.; SILVA, M. D.; CARVALHO, R. Revisão integrativa: o que é e como fazer. Einstein, v.8, n.1, p.1026, 2010.

POMPEO, D. A.; ROSSI, L. A.; GALVÃO, C. M. Revisão integrativa: etapa inicial do processo de validação de diagnóstico de enfermagem. Acta Paul Enferm, v.22, n.4, p.434-8, 2009.

SILVA, C. C. et al. Abordagem por competências no processo ensino aprendizagem. Rev Bras Enferm, v. 58, n. 1, p. 91-4, 2005.
NICHIATA L. Y. I. et al. Relato de uma experiência de ensino de enfermagem em saúde coletiva: a informática no ensino de vigilância epidemiológica. Rev Esc Enferm, v. 37, n.3, p.36-43, 2003

CAMILlO, S.O.; MAIORINO, F. T.; CHAVES, L. C. O ensino de enfermagem sobre HIV/Aids sob a ótica da cidadania. Rev Gaúcha Enferm, v.34, n.3, p.117-123, 2013.

LIMA, T. G. F. M. S.; PORTO, I. S.; FRANCISCO, M.T. R. Teoria e prática docente sobre HIV/AIDS na graduação em enfermagem, Esc. Anna Nery Rev. Enferm, v. 9, n.2, p.247-254, 2005.

MIYASAKI, S. C. S.; BUENO, S. M. V. Pesquisa-Ação sobre sexualidade, DST/AIDS com enfermeiros professores da escola de enfermagem. DST-Jornal brasileiro de doenças sexualmente transmissíveis, v. 13, n. 5, p.46-53, 2001.

HOLANDA, E. R. et al. Tendências na produção do conhecimento científico de enfermagem sobre HIV/AIDS: estudo bibliométrico. Online braz j nurs [ homepage na Internet]. Dec, v.12, n.4, p. 986-97, 2013. Disponível em: http://www.objnursing.uff.br/index.php/nursing/article/vie w/3818http://dx.doi.org/10.5935/1676-4285.20133818.

Acesso em: junho de 2014.

OLIVEIRA, D. C. et al. A produção de conhecimento sobre HIV/AIDS no campo da teoria de representações sociais em 25 anos da epidemia. Rev Eletr Enf, v.9, n.3, p.821-34, 2007.Disponível em: http://www.revistas.ufg.br/index.php/fen/article/view/750 8. Acesso em: 24 de junho de 2014.

MERIGHI, M. A.; BARBOSA, G. R.; FERREIRA, F. C. Estudo bibliométrico sobre dissertações e teses em enfermagem com abordagem fenomenológica: tendência e perspectivas. Rev latino am enferm. Aug, v.15, n.4, p.64550, 2007.

ARAÚJO, M. A L.; ARAUJO, T. L.; DAMASCENO, M. M. C. Conhecimento em HIV/AIDS de 1998 a 2005: estudos publicados em periódicos de Enfermagem. Esc Anna Nery Rev Enferm. Abr, v.10, n.1, p.126-31, 2006.

COFEN.CONSELHO FEDERAL DE ENFERMAGEM. Brasília (DF) 2009. Resolução no 358 de 2009: Dispõe sobre a Sistematização da Assistência de Enfermagem SAE e o Processo de Enfermagem. Disponível em: http://www.portaldaenfermagem.com.br/legislacao_read.a sp?id=337. Acesso em: 23 de junho de 2014 . 